2. J. Dieudonné, Sur un espace localement compact non metrisable, An. Acad. Brasil Ci. vol. 19 (1947) pp. 67-69.

3. C. H. Dowker, On a theorem of Hanner, Ark. Mat. vol. 2 (1952) pp. 307-313.

4. K. Morita and S. Hanai, Closed mappings and metric spaces, Proc. Japan Acad. vol. 32 (1956) pp. 10-14.

5. J. Nagata, On a necessary and sufficient condition of metrizability, J. Inst. Polytech. Osaka City Univ. Ser. A. vol. 1 (1950) pp. 93-100.

6. Yu. M. Smirnov, A necessary and sufficient condition for metrizability of topological spaces, Dokl. Akad. Nauk SSSR vol. 77 (1951) pp. 197-200.

7. - On the metrizability of bicompacts decomposable into a sum of sets with countable basis, Fund. Math. vol. 43 (1956) pp. 387-393.

8. A. H. Stone, Metrizability of decomposition spaces, Proc. Amer. Math. Soc. vol. 7 (1956) pp. 690-700.

The University of Manchester, England

\title{
CORRESPONDANCE ENTRE DEUX SURFACES PAR DES FAISCEAUX DE TANGENTES PARALLÈLES
}

\section{P. DRĂGIL $\breve{~}$}

1. En continuant nos recherches relatives à la correspondance par parallélisme des plans tangents des deux surfaces, nous nous sommes proposé d'établir le nombre maximum possible des couples de tangentes parallèles. D'après les résultats obtenus antérieurement il sembla que ce nombre maximum doit être quatre. Mais nous avons découvert recemment que cela n'est pas ainsi et qu'il existe une catégorie spéciale de correspondances, par une double infinité de couples de tangentes parallèles.

2. Nous notons comme d'habitude les deux surfaces, rapportées au même système de paramètres curvilignes $u, v$, par $S(x, y, z), \bar{S}(\bar{x}, \bar{y}, \bar{z})$ et nous utillisons les notations vectorielles usuelles $r, r_{u}, r_{v}, \cdots$ pour représenter les coordonnées $x, y, z$ et leurs dérivées $\partial x / \partial u, \cdots$, $\partial x / \partial v, \cdots$.

Nous considérons le couple de deux surfaces $S, \bar{S}$, liées par les relations

$$
\begin{aligned}
& \bar{r}_{u}=\lambda r_{u}, \\
& \bar{r}_{v}=\mu r_{v} .
\end{aligned}
$$

Il est aisé de voir que les tangentes aux lignes $u, v$, sur la première

Received by the editors July 2, 1958. 
surface, sont parallèles aux tangentes homologues, aux lignes $u, v$ sur la seconde surface et encore que les plans tangents, aux points correspondants des deux surfaces, sont aussi parallèles.

Cherchons maintenant s'il est possible d'établir une relation entre les coefficients $\lambda$ et $\mu$, de manière que, en déhors de la correspondance par parallélisme linéaire, définie par les équations (1), il soit possible aussi la correspondance par parallélisme ponctuel, et que le nombre des paires des tangentes $r_{s}, r_{t}$, sur la première surface, qui sont parallèles aux tangentes $\bar{r}_{t}, \bar{r}_{s}$, sur la seconde surface, soit le plus grand possible. Faisons pour cela le changement de variables

$$
u=\phi(s, t), \quad v=\psi(s, t)
$$

et écrivons ensuite les équations de condition de la correspondance par parallélisme ponctuel

$$
\frac{\bar{x}_{s}}{x_{t}}=\frac{\bar{y}_{s}}{y_{t}}=\frac{\bar{z}_{s}}{z_{t}}, \quad \frac{\bar{x}_{t}}{x_{s}}=\frac{\bar{y}_{t}}{y_{s}}=\frac{\bar{z}_{t}}{z_{s}} .
$$

En faisant les substitutions (2) et en tenant compte encore des relations (1), il vient

$$
\begin{aligned}
& \frac{\lambda x_{u} u_{s}+\mu x_{v} v_{s}}{x_{u} u_{t}+x_{v} v_{t}}=\frac{\lambda y_{u} u_{s}+\mu y_{v} v_{s}}{y_{u} u_{t}+y_{v} v_{t}}=\frac{\lambda z_{u} u_{s}+\mu z_{v} v_{s}}{z_{u} u_{t}+z_{v} v_{t}}, \\
& \frac{\lambda x_{u} u_{t}+\mu x_{v} v_{t}}{x_{u} u_{s}+x_{v} v_{s}}=\frac{\lambda y_{u} u_{t}+\mu y_{v} v_{t}}{y_{u} u_{s}+y_{v} v_{s}}=\frac{\lambda z_{u} u_{t}+\mu z_{v} v_{t}}{z_{u} u_{s}+z_{v} v_{s}} .
\end{aligned}
$$

Après calculs et réductions nous obtenons le système

$$
\begin{aligned}
& \lambda u_{s} v_{t}-\mu v_{s} u_{t}=0, \\
& \lambda u_{t} v_{s}-\mu v_{t} u_{s}=0,
\end{aligned}
$$

qui peut s'écrire encore sous une autre forme

$$
\begin{aligned}
& (\lambda-\mu)\left(u_{s} v_{t}+v_{s} u_{t}\right)=0, \\
& (\lambda+\mu)\left(u_{s} v_{t}-v_{s} u_{t}\right)=0 .
\end{aligned}
$$

Nous avons donc deux cas à envisager

$$
\begin{aligned}
\lambda-\mu & =0, \\
u_{s} v_{t}-v_{s} u_{t} & =0,
\end{aligned}
$$

ou bien

$$
\begin{aligned}
\lambda+\mu & =0, \\
u_{s} v_{t}+v_{s} u_{t} & =0 .
\end{aligned}
$$


Le premier cas est manifestement impossible, puisque le jacobien $D(u, v) / D(s, t)$ de la transformation doit être différent de zéro.

Dans le second cas il est aisé de voir qu'on peut faire $\infty^{2}$ changements de variables (2), pour lesquels soient satisfaites les relations (4).

Exemple. En prenant

$$
u=s t,
$$

nous trouvons, par l'intégration de l'équation

$$
s v_{s}+t v_{t}=0,
$$

la solution

$$
v=\theta\left(\frac{t}{s}\right)
$$

$\theta$ étant une fonction dérivable arbitraire.

3. Il s'ensuit alors que, sur les deux surfaces $S, \bar{S}$, liées par les équations

$$
\begin{aligned}
& \bar{r}_{u}=\lambda r_{u}, \\
& \bar{r}_{v}=-\lambda r_{v},
\end{aligned}
$$

il y a une infinité de systèmes de coordonnées curvilignes $s, t$, le long desquelles les tangentes $r_{s}, r_{t}, \bar{r}_{s}, \bar{r}_{t}$, se correspondent par parallélisme ponctuel. Il est intéressant de remarquer qu'on peut prendre les mêmes systèmes de coordonnées curvilignes sur toutes les paires de surfaces, $S, \bar{S}$ parallèles, parce qu'ils ne dependent pas du coefficient $\lambda$.

Timisoara, Roumania 